\title{
Risk-Based Two-Stage Stochastic Optimization Problem of Micro-Grid Operation with Renewables and Incentive-Based Demand Response Programs
}

\author{
Pouria Sheikhahmadi ${ }^{1}$, Ramyar Mafakheri ${ }^{2}$, Salah Bahramara ${ }^{2}{ }^{*}$, Maziar Yazdani Damavandi $^{3}$ \\ and João P. S. Catalão ${ }^{3,4,5, *}$ \\ 1 Department of Electrical and Computer Engineering, University of Kurdistan, Sanandaj 66131, Iran; \\ pouria.sheikhahmadi@yahoo.com \\ 2 Department of Electrical Engineering, Sanandaj Branch, Islamic Azad University, Sanandaj 66131, Iran; \\ rami.mafakheri@gmail.com \\ 3 C-MAST, University of Beira Interior, 6201-001 Covilhã, Portugal; maziar.yazdani.d@gmail.com \\ 4 INESC TEC and the Faculty of Engineering of the University of Porto, 4200-465 Porto, Portugal \\ 5 INESC-ID, Instituto Superior Técnico, University of Lisbon, 1049-001 Lisbon, Portugal \\ * Correspondence: s_bahramara@yahoo.com (S.B); catalao@ubi.pt (J.P.S.C.)
}

Received: 17 February 2018; Accepted: 7 March 2018; Published: 9 March 2018

\begin{abstract}
The operation problem of a micro-grid (MG) in grid-connected mode is an optimization one in which the main objective of the MG operator (MGO) is to minimize the operation cost with optimal scheduling of resources and optimal trading energy with the main grid. The MGO can use incentive-based demand response programs (DRPs) to pay an incentive to the consumers to change their demands in the peak hours. Moreover, the MGO forecasts the output power of renewable energy resources (RERs) and models their uncertainties in its problem. In this paper, the operation problem of an MGO is modeled as a risk-based two-stage stochastic optimization problem. To model the uncertainties of RERs, two-stage stochastic programming is considered and conditional value at risk (CVaR) index is used to manage the MGO's risk-level. Moreover, the non-linear economic models of incentive-based DRPs are used by the MGO to change the peak load. The numerical studies are done to investigate the effect of incentive-based DRPs on the operation problem of the MGO. Moreover, to show the effect of the risk-averse parameter on MGO decisions, a sensitivity analysis is carried out.
\end{abstract}

Keywords: demand response programs; micro-grid; renewable energy resources; risk-management; two-stage stochastic programming

\section{Introduction}

\subsection{Motivation and Aim}

Distributed energy resources (DERs) are a suitable solution to address economic, environmental, and technical issues of power systems.

DERs consist of distributed generations (DGs), energy storages (ESs), and demand side management (DSM), which are utilized to meet the demand locally in distribution networks [1]. The MG concept is proposed to operate these resources properly in both grid-connected and stand-alone modes [2,3].

Recently, several studies have been done in the field of MGs, including control and stability, voltage imbalance, fault current limit, the impact of maximum power point tracking (MPPT) of renewable energy resources (RERs), dynamic operation and so on. Dynamic operation and control of MGs are studied in [4]. A designed novel intelligent damping controller (NIDC) is used to improve 
the transient stability in a hybrid power multi-system in [5]. The MPPT of various types of DERs are explained and discussed in [6]. Various fault current analyses of MGs are investigated using novel methods and algorithms in [7,8]. Energy management and optimal operation of MGs using stochastic programming and robust methods are presented to control RER uncertainties in $[9,10]$. Connection decisions of transformers are done using a modified optimization approach in [11]. Regarding the mentioned subjects related to the MG studies, one of the main problems of MGs is to meet its demand with minimum cost during the operation period with optimal scheduling of its local resources and optimal energy trading with the main grid in the grid-connected mode. This problem is modeled from the viewpoint of the MG operator (MGO) as an optimization problem.

In this context, demand response programs (DRPs) are suitable tools for the MGO to decrease the demand in the peak hours and minimizing its operation cost. DRPs are divided into the incentive-based and the time-based programs which of which detailed description are presented in [12]. The MGO can use the incentive-based DRPs to pay incentives to the consumers to reduce or cut their consumptions in the peak hours. On the other hand, MG loads (MGLs) consist of inelastic loads and elastic (controllable) ones. Controllable loads can participate in these DRPs to decrease or shift their consumption to achieve the revenue from these programs.

Due to the high penetration of RERs and uncertainties of their output power, the MGO faces a high risk in its operation problem. The aim of this paper is to model the operation problem of an MG considering the different non-linear economic models of DRPs as well as the uncertainties of RERs. For this purpose, the problem is modeled as a risk-based two-stage stochastic optimization one which uses the conditional value at risk (CVaR) index to control the uncertainties as well as their impacts on the MGO decisions.

\subsection{Literature Review and Contributions}

The operation problem of MGs in the presence of DRPs and RERs is investigated in many studies. In [13], an optimal scheduling of an MG consisting of wind turbine (WT), photo-voltaic (PV) array, and ES is proposed to minimize the operation cost while the technical constraints are satisfied. The impact of different weather conditions on the operation cost of a renewable energy-based MG is investigated in [14]. The MG's operation problem is modeled considering RERs and incentive-based DRP in [15] in which the main objective of the MGO is to minimize the fuel cost of DGs with DRPs. Energy management of an MG consisting of PV, fuel cell (FC), and battery is discussed in [16]. The operation problem of MGs is investigated using deterministic approaches in [13-16] in which the uncertain parameters are not modeled in the problems.

In [17], the probabilistic approach is used to model the energy management of an MG considering uncertainties of demand, energy price, wind speed, and solar radiation. Time series data is employed to model the uncertainties of wind speed and demand in the operation problem of MGs. The operation problem of an MG in stand-alone mode is modeled using probabilistic approach in [18]. Moreover, in [19], to mitigate the impact of RER's uncertainties, a novel approach to optimal energy and reserve scheduling of DGs and ESs is proposed. In [20], the stochastic approach is used to model the operation problem of an MG consisting of WT, PV, ES, and responsive loads. The DRPs and RERs are modeled in the operation problem of an MG considering the uncertainties of wind speed and solar radiation [21]. The uncertainties of WT, PV, and load are modeled in the operation problem of an MG using interval optimization approach in [22]. Although the MGO faces high risks in decision making in the presence of RERs, the appropriate models are not proposed to deal with these uncertainties in the literature. For this purpose, in this paper, a two-stage stochastic optimization approach is proposed to model the RERs uncertainties. The CVaR index is applied to the proposed model to control the uncertainties as well as the MGO's risk-level in the operation decisions.

The non-linear economic models of the time-based DRPs are proposed in [23] and they are extended in [24] as the form of the incentive-based DRPs to apply to the economic dispatch problem. It should be noted that the mentioned DRPs models have been not applied to the operation problem 
of MGs. Therefore, in this paper, the operation problem of an MG consisting of RERs, DGs, and ES is modeled considering the non-linear economic models of incentive-based DRPs. The non-linear economic models of the time-based DRPs in [23] are developed to model the incentive-based DRPs in the operation problem of MGs.

Therefore, considering the MG operation models proposed in the literature and described in Table 1, the main contributions of this paper are as follows:

1. Modeling the operation problem of an MG considering the non-linear economic models of the incentive-based DRPs and uncertainties of RERs as a risk-based two-stage optimization problem.

2. Control the effects of RERs uncertainties on the optimal decisions of the MGO using the CVaR index.

Table 1. Comparison between the micro-grid (MG) operation models reported in the literature.

\begin{tabular}{cccccc}
\hline Ref. & MG's Resources & The Type of Model & $\begin{array}{c}\text { Demand } \\
\text { Response Model }\end{array}$ & Risk Management & Approach \\
\hline$[1]$ & PV, WT, DG, ES, DR & Probabilistic & Load shifting (linear) & No & $\begin{array}{c}\text { Mixed Integer Linear } \\
\text { Programming (MILP) }\end{array}$ \\
\hline$[2]$ & PV, WT, DG, ES & Probabilistic & - & No & MILP \\
\hline$[3]$ & PV, WT, DG, ES, DR & One-stage stochastic & $\begin{array}{c}\text { Interruptible load } \\
\text { (linear) }\end{array}$ & Yes & MILP \\
\hline$[14]$ & PV, DG, ES & Deterministic & - & No & $\begin{array}{c}\text { Non-Linear } \\
\text { Programming (NLP) }\end{array}$ \\
\hline$[15]$ & PV, WT, DG, DR & Deterministic & $\begin{array}{c}\text { Incentive-based } \\
\text { (linear) }\end{array}$ & No & NLP \\
\hline$[16]$ & PV, DG, ES & Deterministic & - & No & MILP \\
\hline$[17]$ & $\begin{array}{c}\text { PV, WT, DG ES } \\
\text { (stand alone MG) }\end{array}$ & Probabilistic & - & No & NLP \\
\hline$[18]$ & Load shedding & One-stage stochastic & - & Yes & MILP \\
\hline$[20]$ & PV, WT, DG, ES, DR & Probabilistic & Price-based (linear) & No & NLP \\
\hline$[21]$ & PV, WT, DG, ES, DR & Probabilistic & $\begin{array}{c}\text { Incentive-based } \\
\text { payments as price offer } \\
\text { packages (linear) }\end{array}$ & No & Yes \\
\hline This paper & PV, WT, DG, ES, DR & Two-stage stochastic & $\begin{array}{c}\text { Incentive-based } \\
\text { (linear and non-linear) }\end{array}$ & MILP/MINLP \\
\hline
\end{tabular}

The rest of the paper is organized as follows. Section 2 describes the problem description. The problem is formulated in Section 3. The numerical results are represented in Section 4 and the conclusion is given in Section 5.

\section{Problem Description}

The MG consists of PV array, WT, micro-turbine (MT), FC, and ES, with all these resources connected to the low voltage network as shown in Figure 1. Moreover, the MG is connected to the distribution network via a distribution transformer to make energy interact with distribution company (Disco). Disco participates in the wholesale energy market to purchase its required energy. For this purpose, when Disco acts as a price-taker player in the day-ahead market, it forecasts the market prices for the next day using different forecasting methods reported in detail in the literature. Then, Disco determines the price of trading energy with the MG as the percent of these forecasted prices considering the power losses of the transmission and distribution networks and its profit. Regarding these prices, the MGO schedules its power resources and decides about purchased power from Disco since the main objective of the MGO is to supply the MGL with minimum costs as shown in Figure 2. Then Disco purchases this required energy from the market. This process is done in the previous day of the real operation and the results are sent to the power system and the distribution system operators. The power system and the distribution system operators are responsible for meeting the required energy of Disco and the MG, respectively. In the proposed model, the economic viewpoint 
of Disco is considered. To model the technical and environmental aspects of Disco, Disco will be considered responsible for supplying the demand of the MG with optimal scheduling of DERs and purchasing power from the market. Also, in the presence of the RERs in the distribution network, Disco purchases lower energy from the market to meet the demand of the MG and other demand consumption of consumers.

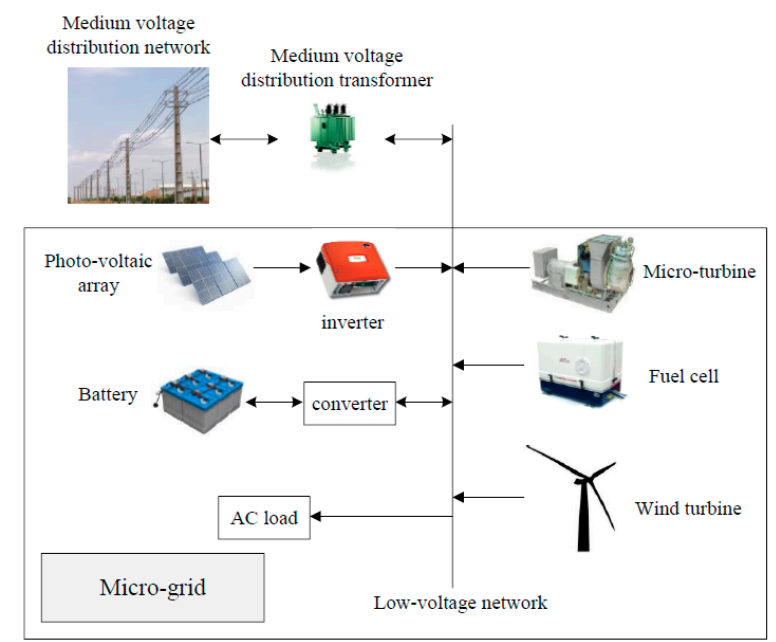

Figure 1. Configuration of the proposed micro-grid.

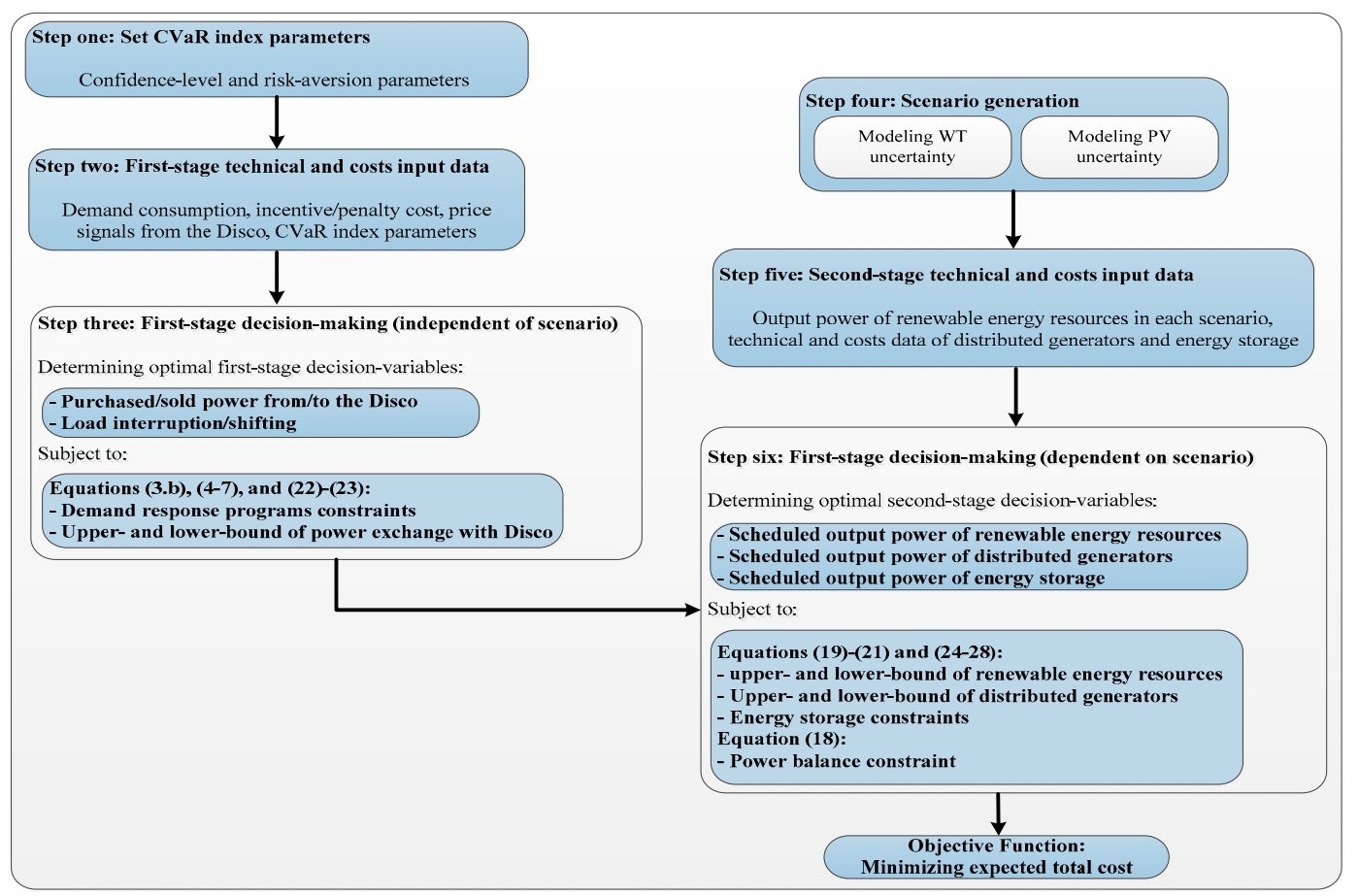

Figure 2. The decision making framework of MGO.

To model the uncertainties of the output power of WT and PV array, the operation problem of the MGO is modeled as a risk-based two-stage stochastic optimization problem as shown in Figure 2. In this problem, the decision variables of the first-stage problem are power exchange with Disco and DRPs, which are independent from scenarios. The decision variables of the second-stage problem are output power of RERs and DGs and power charging/discharging of the ES, which depend on scenarios; the MGO decides about these variables in each scenario based on the optimal amount of the first-stage 
decision variables. In this context, the MGO can mitigate the impact of the RERs' uncertainties and manage its risk by changes the amount of decision variables in the first-stage decisions. Moreover, the MGO with high risk prefers the second-stage decisions to achieve a lower total operation cost. The proposed framework for the MGO which is shown in Figure 2 is modeled as a mathematical optimization problem in the next section.

\section{Mathematical Modeling}

In this section, mathematical modeling of the operation problem of the grid-connected MG is presented in detail as follows.

\subsection{Assumptions}

Two assumptions are considered in this model as follows:

- The RERs' uncertainties are only modeled in the MG operation problem and modeling the equipment failures; the factors that make interrupt the MG connection to the distribution network are beyond the scope of this paper.

- It is assumed that the end users are equipped with smart meters and the appropriate communication infrastructure is designed between the MGO and end-users to implement the DRPs.

\subsection{Demand Response Models (DRMs)}

The demand response mechanism used in this paper is an interruptible/curtailable (I/C) service as one of the incentive-based demand response programs described in [4]. To consider responsive loads at the customer's side, the elasticity is defined as the sensitivity of responsive loads to the price in Equations (1)-(3) as follows:

$$
\text { Elasticity }=\frac{\partial D / D^{\text {initial }}}{\partial \pi / \pi^{\text {initial }}}=\frac{\partial D}{\partial \pi} \frac{\pi^{\text {initial }}}{D^{\text {initial }}}
$$

where $D^{\text {initial }}, \pi^{\text {initial }}$ are the initial load and initial price before defining a new price, and $D$ is the demand level after introducing the new price and $\pi$ is the new price.

$$
\text { Elasticity } t_{t, t}=\frac{D_{t}^{\mathrm{DRM}}-D_{t}^{\text {initial }}}{\pi_{t}^{\text {PBDR }}-\pi_{t}^{\text {initial }}} \times \frac{\pi_{t}^{\text {initial }}}{D_{t}^{\text {initial }}} \leq 0
$$

Since some loads such as lighting have single period sensitivity, they are not transferred to the other time periods and they are defined as "self-elasticity" and have negative values. Equation (2) is the self-elasticity at time $t$.

$$
\text { Elasticity }_{t, t^{\prime}}=\frac{D_{t}^{\mathrm{DRM}}-D_{t}^{\text {initial }}}{\pi_{t^{\prime}}^{P B D R}-\pi_{t^{\prime}}^{\text {initial }}} \times \frac{\pi_{t^{\prime}}^{\text {initial }}}{D_{t}^{\text {initial }}} \geq 0
$$

Other loads can be transferred to other periods and have multi-period sensitivity, hence, they are called "cross-elasticity" and they have positive values. Equation (3) is the cross elasticity between time $t$ and $t^{\prime}$, where $D_{t}^{D R M}$ and $\pi_{t}^{P B D R}$ are the demand level and the new electricity price after implementation of DRPs.

According to Equations (4)-(7), the cross-elasticity defined in Equation (3) is used to determine the amount of the MG's demand after implementing each type of the DR model. The economic models 
of responsive loads are presented in four types including linear, power, exponential, and logarithmic, respectively as follows:

$$
\begin{gathered}
D_{t}^{\text {DRM-lin }}=D_{t}^{\text {initial }} \times\left(1+\sum_{t \prime} \frac{\pi_{t^{\prime}}^{P B D R}-\pi_{t^{\prime}}^{\text {initial }}+A_{t^{\prime}}+\text { pen }_{t^{\prime}}}{\pi_{t^{\prime}}^{\text {initial }}} \times \text { Elasticity }_{t, t^{\prime}}\right) \quad \forall t \\
D_{t}^{\text {DRM-Pow }}=\left(D_{t}^{\text {initial }} \times \prod_{t^{\prime}}\left(\frac{\pi_{t^{\prime}}^{\text {PBDR }}+A_{t^{\prime}}+p e n_{t^{\prime}}}{\pi_{t^{\prime}}^{\text {initial }}}\right) \text { Elasticity }_{t, t^{\prime}}\right) \quad \forall t \\
D_{t}^{\text {DRM-EXP }}=D_{t}^{\text {initial }} \times \operatorname{Exp}\left(\sum_{t^{\prime}} \frac{\pi_{t^{\prime}}^{P B D R}-\pi_{t^{\prime}}^{\text {initial }}+A_{t^{\prime}}+p e n_{t^{\prime}}}{\pi_{t^{\prime}}^{\text {initial }}} \times \text { Elasticity }_{t, t^{\prime}}\right) \quad \forall t \\
D_{t}^{\text {DRM-Log }}=D_{t}^{\text {initial }} \times\left(1+\sum_{t^{\prime}}\left(\ln \left(\frac{\pi_{t^{\prime}}^{\text {PBDR }}+A_{t^{\prime}}+p e n_{t^{\prime}}}{\pi_{t^{\prime}}^{\text {initial }}}\right)\right) \times \text { Elasticity }_{t, t^{\prime}}\right) \quad \forall t
\end{gathered}
$$

\subsection{Modeling Uncertainties}

To model the uncertainties of the output power of RERs, the Weibull and irradiance distribution models are used as probability distribution functions (PDFs) of wind speed and solar radiation, respectively. Then, 10,000 scenarios are generated and are reduced to 15 scenarios using the General Algebraic Modeling System/Scenario Reduction (GAMS/SCENRED) package (24.1.2, GAMS Development Corporation, Washington, DC, USA) and the fast-forward scenario reduction technique. These scenarios determine the stochastic output power of the WTs and PVs and are considered as the operation scenarios. Each scenario consists of wind speed and solar radiation data for the time period of operation. Then, the output power of RERs is calculated in each scenario as presented in the next sub-section.

\subsection{RERs Model}

The output power of WT is calculated using the wind speed in each scenario, the WT's power curve, and other characteristics of the WT. According to references [25,26], to model wind speed behavior, a Rayleigh distribution function is used. In Weibull distribution when the shape index is equal to 2, it changes to the Rayleigh distribution. For specific wind energy system, the output power is defined in [21].

PV cells convert the solar irradiance energy to electrical energy, that the output power of them in each scenario depends on the number of cells, direction of cells, temperature and weather conditions which are given in [21].

\subsection{Operation Cost}

The expected total cost (ETC) and the total cost of the MG in each scenario is given as follows:

$$
\begin{gathered}
\mathrm{ETC}=\sum_{s=1}^{S} \rho_{s} \cos t_{s} \\
\operatorname{cost}_{s}=\sum_{t=1}^{T}\left[\sum_{g=1}^{G} C_{g}^{\mathrm{DG}} P_{g, t, s}^{\mathrm{DG}}+C_{t}^{g r i d-i n} P_{t}^{g r i d-i n}-C_{t}^{\text {grid-out }} P_{t}^{g r i d-o u t}+C^{\mathrm{ES}} P_{t, s}^{\mathrm{ES}}+S\left(D_{t}^{\mathrm{DRM}}\right)\right] \quad \forall s
\end{gathered}
$$

The first term of Equation (9) is the operation cost of DGs. The second and third terms model the cost/revenue of purchasing/selling electrical energy from/to Disco, and the fourth term represents 
the operation cost of the ES. Since the operation cost of RERs is very low, they are not modeled in the operation cost. The last term is the cost of DRPs consisting of three parts as follows:

$$
\begin{gathered}
S\left(D_{t}^{\mathrm{DRM}}\right)=S_{1}\left(D_{t}^{\mathrm{DRM}}\right)+S_{2}\left(D_{t}^{\mathrm{DRM}}\right)+S_{3}\left(D_{t}^{\mathrm{DRM}}\right) \quad \forall t \\
S_{1}\left(D_{t}^{\mathrm{DRM}}\right)=-\pi_{t}^{\text {PBDR }} D_{t}^{\mathrm{DRM}} \quad \forall t \\
S_{2}\left(D_{t}^{\mathrm{DRM}}\right)=A_{t}\left(D_{t}^{\text {initial }}-D_{t}^{\mathrm{DRM}}\right) \quad \forall t \\
S_{3}\left(D_{t}^{\mathrm{DRM}}\right)=\operatorname{pen}_{t}\left(\left(D_{t}^{\text {initial }}-D_{t}^{\mathrm{DRM}}\right)-D_{t}^{\text {contract }}\right) \quad \forall t
\end{gathered}
$$

The first term of Equation (10) is related to the revenue from selling power to the MGL after implementation of DRPs considering different DRMs. The second term is used to model the total payment to the responsive MGL due to its participation in voluntary incentive-based DRPs in peak period. The MGO contracts with end-users which participate in incentive-based DRPs in a previous day before the real operation. In the real operation, the energy consumptions by end users in each hour are measured by smart meters and are sent to the MGO via a designed communication system. Then, the difference between the initial demand $\left(D_{t}^{\text {initial }}\right)$ and the demand after implementation of DR $\left(D_{t}^{\mathrm{DRM}}\right)$ is compared with the amount of demand assigned in the contract to be curtailed $\left(D_{t}^{\text {contract }}\right)$ and the third term is used to model the total penalty of customers that participate in forced incentive-based DRPs and the responsive MGL penalized due to not decreasing their loads.

\subsection{Risk Management}

In this paper, the CVaR index is used to control the impact of uncertain parameters on the operation problem of the MGO. The CVaR at the $\alpha$ confidence level ( $\alpha$-CVaR) can be defined as the expected cost in $(1-\alpha) \times 100 \%$ worst scenarios as follows [27]:

$$
\begin{gathered}
\mathrm{CVaR}=\delta-\frac{1}{1-\alpha} \sum_{S=1}^{N S} \rho_{s} \lambda_{s} \\
\lambda_{s} \geq \delta-\operatorname{cost}_{s} \quad \forall s \\
\lambda_{s} \geq 0 \quad \forall s
\end{gathered}
$$

where $\delta, \alpha, N_{s}, \rho_{s}$, and $\lambda_{s}$ are the value at risk, confidence level, number of scenarios, probability of each scenario, and an auxiliary positive variable equal to value at risk minus cost at each scenario, respectively.

\subsection{Objective Function}

In this paper, the decision-making framework of the MGO is modeled as a risk-based two stage stochastic model. The objective function of the model is to minimize the ETC and the expected cost of $(1-\alpha \%)$ of the worst scenarios. The objective function is as follows:

$$
\text { Minimize } \sum_{s=1}^{S} \rho_{s} \operatorname{cost}_{s}+\beta \mathrm{CVaR}
$$

where $\beta$ is the risk aversion parameter. The MGO is risky when $\beta$ is equal to zero and with increasing $\beta$, the MGO becomes a more risk-averse player. 


\subsection{Equality and Inequality Constraints}

Power Balance Constraint

The power balance between MG's energy resources and the MGL is defined as follows:

$$
\sum_{g}^{G} P_{g, t, s}^{\mathrm{DG}}+P_{t, s}^{\mathrm{WT}}+P_{t, s}^{\mathrm{PV}}+P_{t}^{g r i d-i n}+P_{t, s}^{\mathrm{ES}}=D_{t}^{\mathrm{DRM}}+P_{t}^{\text {grid-out }} \quad \forall t, s
$$

Upper/Lower Output Power Limits of the DGs:

$$
\underline{P}_{g}^{\mathrm{DG}} \leq P_{g, t, s}^{\mathrm{DG}} \leq \bar{P}_{g}^{\mathrm{DG}} \quad \forall g, t, s
$$

Minimum/Maximum Output Power of WTs:

$$
0 \leq P_{t, s}^{\mathrm{WT}} \leq \bar{P}_{t, s}^{\mathrm{WT}} \quad \forall t, s
$$

Maximum/Minimum Output Power of PVs:

$$
0 \leq P_{t, s}^{\mathrm{PV}} \leq \bar{P}_{t, s}^{\mathrm{PV}} \quad \forall t, s
$$

Constraints (20) and (21) are used to limit the upper bounds of the output power of the RERs (i.e., $P V$ and $W T$ ). The $\mathrm{MGO}$ uses these resources regarding its risk-aversion parameter.

Power Exchange Limits between the MG and the Main Grid:

$$
\begin{gathered}
0 \leq P_{t}^{\text {grid-in }} \leq \bar{P}^{\text {grid-in }} u_{t}^{\text {grid }} \quad \forall t \\
0 \leq P_{t}^{\text {grid-out }} \leq \bar{P}^{\text {grid-out }}\left(1-u_{t}^{\text {grid }}\right) \quad \forall t
\end{gathered}
$$

The Energy Storage Constraints:

$$
\begin{gathered}
P_{t, s}^{\mathrm{ES}}=P_{t, s}^{\mathrm{ES}-\text { charge }}+P_{t, s}^{\mathrm{ES}-\text { dicharge }} \quad \forall t, s \\
0 \leq P_{t, s}^{\mathrm{ES}-\text { charge }} \leq \bar{P}_{t, s}^{\mathrm{ES}-\text { charge }} u_{t, s}^{\mathrm{ES}} \quad \forall t, s \\
0 \leq P_{t, s}^{\mathrm{ES}-\text { discharge }} \leq \bar{P}_{t, s}^{\mathrm{ES}-\text { discharge }}\left(1-u_{t, s}^{\mathrm{ES}}\right) \quad \forall t, s \\
\underline{E}^{\mathrm{ES}} \leq E_{t, s}^{\mathrm{ES}} \leq \bar{E}^{\mathrm{ES}} \quad \forall t, s \\
E_{t . s}^{\mathrm{ES}}=E_{t-1 . s}^{\mathrm{ES}}+\eta^{\mathrm{ES}} P_{t, s}^{\mathrm{ES}-\text { charge }}-\frac{P_{t, s}^{\mathrm{ES}-\text { discharge }}}{\eta^{\mathrm{ES}}} \quad \forall t, s
\end{gathered}
$$

where Equation (24) is the output power of ES consisting of power charging/discharging, Equations (25) and (26) are the limitations of power charging/discharging of $E S$, and $u_{t, s}^{E S}$ is a binary variable which shows that the ES cannot charge and discharge in time step $t$ and scenario s, simultaneously. Energy stored in ES and its limitations are defined in Equations (27) and (28).

\subsection{The Limitation of the Adopted Model}

The above model has the following limitations:

- The MG equipment failure rate and its uncertainties are not considered.

- The proposed scenario generation approach only used to model the uncertainties of RERs and cannot model the uncertainties of parameters with unknown PDF. 
The operation problem of the MG is modeled as a mixed-integer linear program (MILP) considering the linear model of DR and as mixed-integer non-linear programs (MINLP) considering the other types of DR models. The model is implemented in GAMS environment and solved by CPLEX and SCIP solvers on a Pentium IV, 2.6-GHz Core i7 with 6GB RAM.

\section{Numerical Results}

\subsection{Input Data}

The operation problem time period is $24 \mathrm{~h}$. The forecasted initial MGL and the output power of WT and PV are given in Figures 3-5, respectively [28]. The output power data of the RERs consisting of the forecasted data and the stochastic output power in each scenario are shown in Figures 4 and 5. The operation problem of the MG is solved by MGO in 15 scenarios regarding the stochastic output power of the RERs.

Disco forecasts electricity prices of the wholesale day-ahead energy market and sends the prices of trading energy to the MGO. Then, the MGO decides on the purchased power from Disco according to these prices. The electricity prices of a sample day which is extracted from Pennsylvania-Jersey-Maryland (PJM) market is considered as the forecasted prices by Disco as shown in Figure 6 [21].

Two types of conventional DGs consisting of FC and MT are considered as MG's DGs. Characteristics of these DGs, characteristics of the ES and other technical data consisting of the capacity of the MG responsive load, the price and the maximum amount of the power exchange between the MG and Disco, and the incentive/penalty payment/cost of the DRPs are given in Table 2. The maximum capacity of the distribution transformer is $300 \mathrm{~kW}$. If the maximum energy consumed by a residential consumer is considered equal to five kilowatts, this MG can meet the energy consumption of approximately 60 residential consumer. Moreover, the self- and cross-elasticity related to DRMs are represented in Table 3 [4].

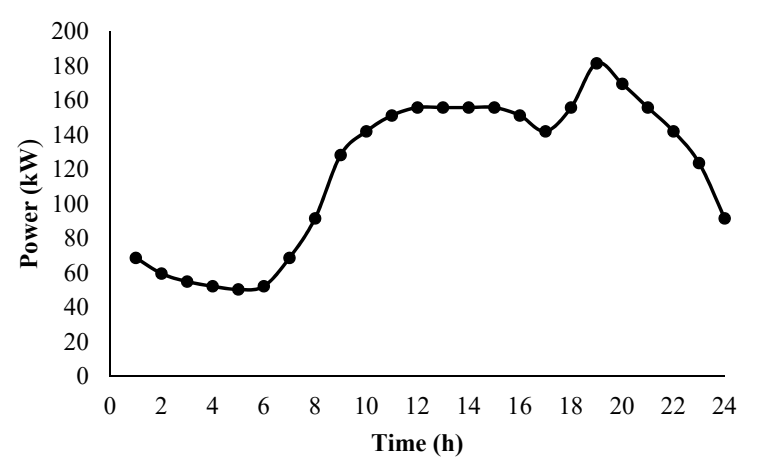

Figure 3. Forecasted MG load consumption.

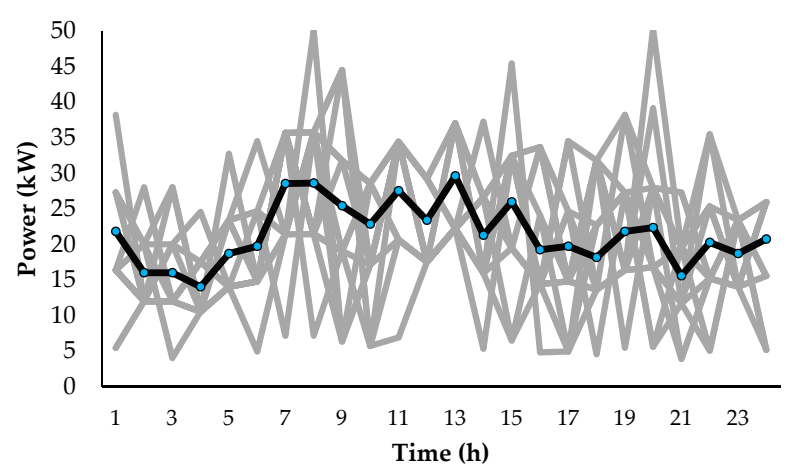

Figure 4. Forecasted and stochastic WT output power. 


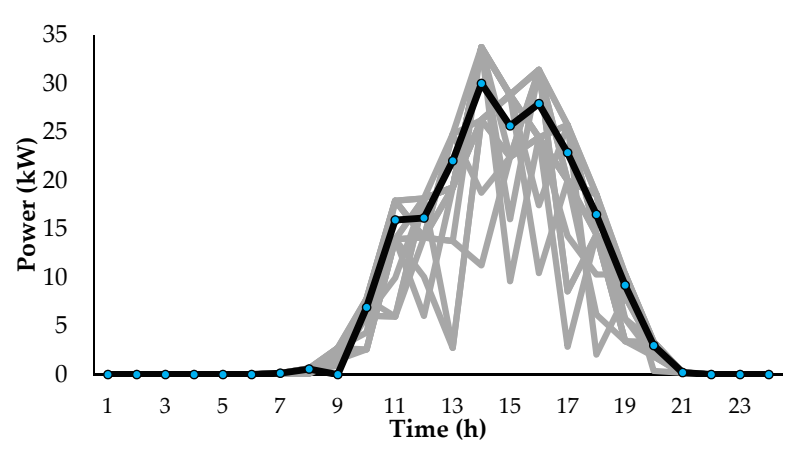

Figure 5. Forecasted and stochastic PV output power.

Table 2. Characteristics of the DGs, ES and other MG technical data.

\begin{tabular}{|c|c|c|c|c|c|c|}
\hline Type of DG & \multicolumn{2}{|c|}{ Marginal Cost $(\mathrm{c} / \mathrm{kWh})$} & $P_{\min }(\mathrm{kW})$ & \multicolumn{3}{|c|}{$P_{\max }(\mathrm{kW})$} \\
\hline MT & \multicolumn{2}{|l|}{4.67} & 6.00 & \multicolumn{3}{|c|}{60.0} \\
\hline FC & \multicolumn{2}{|l|}{3.40} & 3.00 & \multicolumn{3}{|c|}{60.0} \\
\hline Number of ES & Operation cost $(\alpha / k W h)$ & $\underline{E}^{\mathrm{ES}}(\mathbf{k W h})$ & $\bar{E}^{\mathrm{ES}}(\mathbf{k W h})$ & $\bar{P}_{t, s}^{\mathrm{ES}-\text { charge }} / \bar{P}_{t, s}^{\mathrm{ES}-}$ & scharge $(\mathbf{k W})$ & $\eta^{E S}$ \\
\hline 1 & 2 & 9 & 50 & 30 & & 0.95 \\
\hline $\begin{array}{c}\bar{P}^{\text {grid-in }} / \bar{P}^{\text {grid-out }} \\
(\mathbf{k W})\end{array}$ & $\begin{array}{l}\text { Capacity of the MG } \\
\text { responsive loads }\end{array}$ & & ${ }^{-i n} / C_{t}^{\text {grid- }}$ & $(\mathrm{c} / \mathrm{kWh})$ & $\begin{array}{r}\text { The incen } \\
\text { paym }\end{array}$ & $\begin{array}{l}\text { penalty } \\
\text { ost }\end{array}$ \\
\hline 300 & $30 \%$ MG load (MGL) & 1.2 of the & $\begin{array}{r}\text { recasted electr } \\
\text { by Discc }\end{array}$ & ity market prices & $30 \%\left(C_{t}^{g r i c}\right.$ & id-out $)$ \\
\hline
\end{tabular}

Table 3. Self and cross elasticity related to DRMs.

\begin{tabular}{cccc}
\hline & Peak & Off-Peak & Low Load \\
\hline Peak & -0.100 & 0.016 & 0.012 \\
Off-peak & 0.016 & -0.100 & 0.010 \\
Low load & 0.012 & 0.010 & -0.100 \\
\hline
\end{tabular}

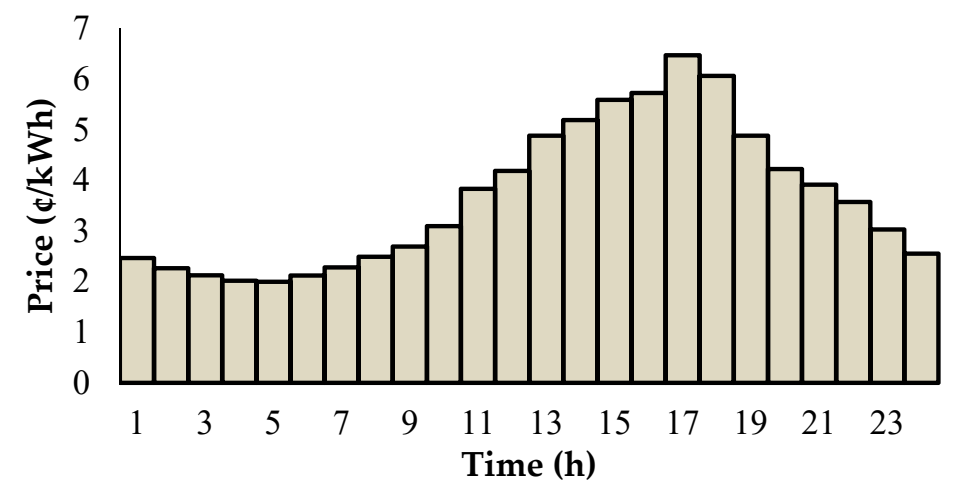

Figure 6. The forecasted electricity market prices by Disco.

\subsection{Case Studies}

The initial demand and the load profile of the MG after applying various DRMs are shown in Figure 7. According to the results, the linear model has the highest and the power model has the lowest load reduction among the proposed four DRP models.

Other results related to the impact of the various types of the DRMs on the MG's decision variables are given in Table 4. The first-stage decisions of the MGO are changed in the presence of DRPs. In fact, the first-stage decisions consisting of the power trading with the main grid (Disco) and load reduction are different in the four types of DR models. According to Table 4, in the DR power model, since the 
less load reduction is occurred, the purchased power from the main grid by the MGO is increased and the power sold to the main grid is decreased. Therefore, its revenue from implementation of DR power model is increased and also its ETC is greater than the other DR models. On the other hand, since the highest load reduction occurred in the linear model, the highest sold power to the main grid is obtained in this model. In other words, using the highest amount of load reduction due to paying the incentive cost to the MG's demand by the MGO does not necessarily guarantee the highest profit for the MGO.

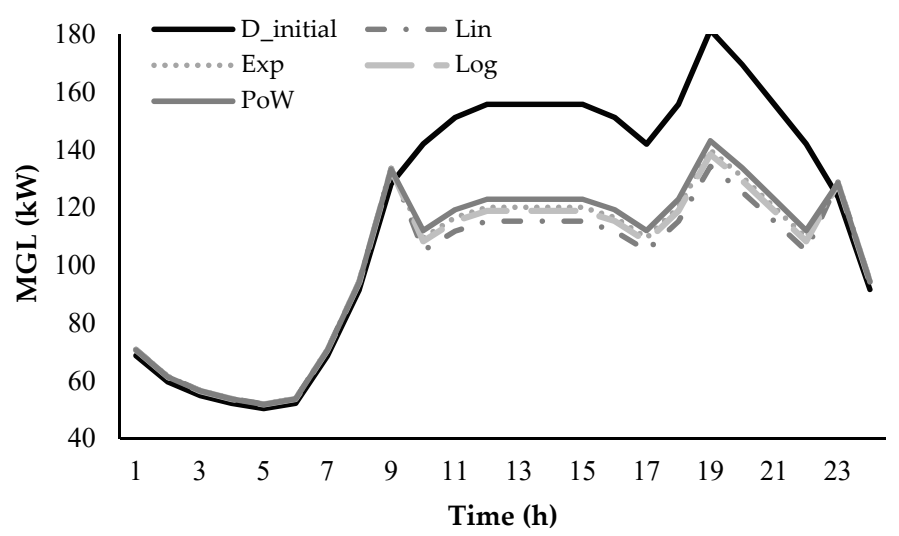

Figure 7. MG load consumption without/with using DRMs.

Table 4. Output results of the MG operation problem in the fourth scenario.

\begin{tabular}{ccccc}
\hline & Linear Model & Power Model & Exponential Model & Logarithmic Model \\
\hline$\sum_{t} P_{t}^{\text {grid-in }}(\mathrm{kW})$ & 642.650 & 677.780 & 666.785 & 657.579 \\
$\sum_{t} P_{t}^{\text {grid-out }}(\mathrm{kW})$ & 352.385 & 290.973 & 313.450 & 323.568 \\
$\sum_{t} P_{t, s}^{\mathrm{WT}}(\mathrm{kW})$ & 502.170 & 502.170 & 502.170 & 502.170 \\
$\sum_{t} P_{t, s}^{\mathrm{PV}}(\mathrm{kW})$ & 174.372 & 174.372 & 174.372 & 174.372 \\
$\sum_{t, g}^{t} P_{g, t, s}^{\mathrm{DG}}(\mathrm{kW})$ & 1322.038 & 1322.038 & 1322.038 & 1322.038 \\
$\sum_{t} P_{t, s}^{\mathrm{ES}-\text { charge }}(\mathrm{kW})$ & 81.916 & 81.916 & 81.916 & 81.916 \\
$\sum_{t} P_{t, s}^{\mathrm{ES}-\text { dcharge }}(\mathrm{kW})$ & 8.474 & 8.474 & 8.474 & 8.474 \\
Total load reduction $(\mathrm{kW})$ & 494.913 & 398.37 & 431.842 & 451.167 \\
Expected total cost $(\mathrm{ETC})(\phi)$ & -43.351 & -43.631 & -43.528 & -43.482 \\
$\sum_{t} s\left(D_{t}^{\mathrm{DRM}}\right)(\phi)$ & -90.157 & -95.724 & -93.733 & -92.730 \\
\hline
\end{tabular}

The ETC of the MGO is presented in Table 5 wherein four types of DRP models and two conditions-with and without applying DRPs on the proposed model-are considered. As shown in this table, the ETC increases considering DRP in the operation problem of the MGO and also between different types of the DR models; the ETC in the DR power model has the highest profit for the MGO.

Table 5. Sensitivity of ETC to the DRP for each type of DRM.

\begin{tabular}{cccccc}
\hline \multicolumn{2}{c}{ DRMs } & Linear Model & Power Model & Exponential Model & Logarithmic Model \\
\hline \multirow{2}{*}{ ETC $(ф)$ Without DR } & -42.618 & -42.618 & -42.618 & -42.618 \\
& With DR & -43.351 & -43.631 & -43.528 & -43.482 \\
\hline
\end{tabular}

The total operation cost of the MGO in all scenarios in the DR power model is shown in Table 6. As mentioned, $(1-\alpha) \%$ of the scenarios are defined as the worst scenarios. In this paper, regarding 
the amount of the confidence-level $(\alpha)$ which is equal to 0.8 , three scenarios can be named as the worst ones (scenarios 2, 3 and 8).

Table 6. Total cost of MG in each scenario in DR power model.

\begin{tabular}{cccccc}
\hline \# Scenario & $\mathbf{1}$ & $\mathbf{2}$ & $\mathbf{3}$ & $\mathbf{4}$ & $\mathbf{5}$ \\
\hline Total cost $(\phi)$ & -43.054 & -41.405 & -42.057 & -43.666 & -43.222 \\
\# Scenario & 6 & 7 & 8 & 9 & 10 \\
Total cost $(\phi)$ & -44.741 & -46.351 & -41.814 & -42.663 & -43.642 \\
\# Scenario & 11 & 12 & 13 & 14 & 15 \\
Total cost $(\phi)$ & -43.019 & -44.558 & -45.897 & -42.907 & -43.087 \\
\hline
\end{tabular}

The power balance of the MG in the DR power model is shown in Figure 8. In hours 12-19 with high energy prices, the purchased power from the main grid by the MGO is zero. Moreover, due to load reduction in these hours and decreasing the demand of MG, the power generation by conventional DGs increases and the MGO sells energy to the main grid. On the other hand, in hours 1-10, 23, and 24 with low energy prices, the purchased power by the main grid increases.

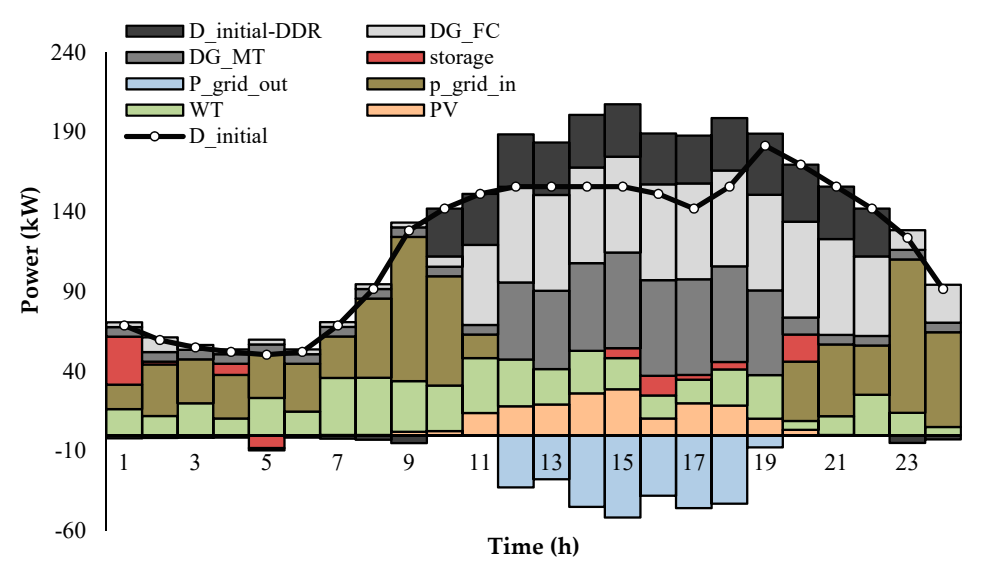

Figure 8. Share of power resources to supply MGL (DR power model).

\subsection{Sensitivity Analysis}

The effect of the risk parameter on the objective function of the MGO in different models of DRP is investigated and the results are shown in Figure 9. In all models, when the risk parameter is increased, the ETC is increased (minus ETC is decreased) and the CVaR is decreased (minus CVaR is increased). This behavior of the MGO occurs because when the risk parameter increases, the MGO is more risk averse and thus its CVaR is decreased.

The effect of the risk-aversion parameter on the optimal decision making of the MGO in different DRMs is given in Table 7. In other words, the risk-averse $(\beta>0)$ MGO changes its first-stage decisions with the aim of achieving less $\mathrm{CVaR}$ (expected cost of the worst scenarios) through controlling the results of the MG operation problem in scenarios 2,3, and 8 . When the value of the risk-aversion parameter increases, the MGO increases the purchased power from the main grid and decreases the sold power to the grid. On the other hand, the utilization of DERs is increased so that the power generation of DGs and power charging/discharging is increased. However, due to uncertainties of WT and PV, utilization from this resource is decreased. Therefore, the results show that when the risk parameter increases, the MGO is more risk averse and so its ETC is decreased in all DRMs. It should be noted that the decision variables related to the amount of the power resources in Table 7 represent the total value in the $24 \mathrm{~h}$ of the fourth scenario and the ETC represents the expected value in the $24 \mathrm{~h}$ of all scenarios. 


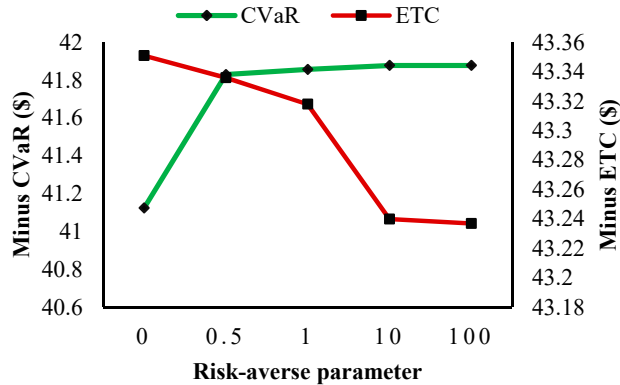

(a)

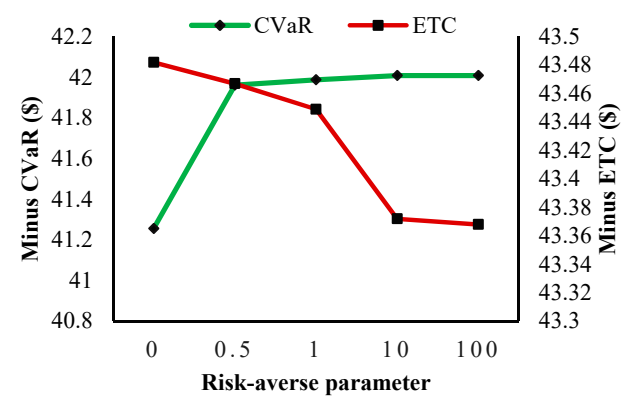

(c)

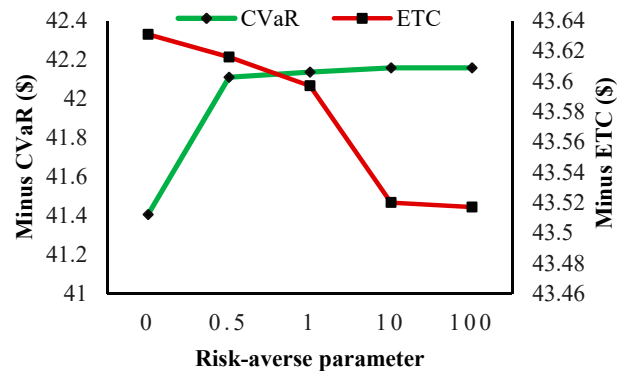

(b)

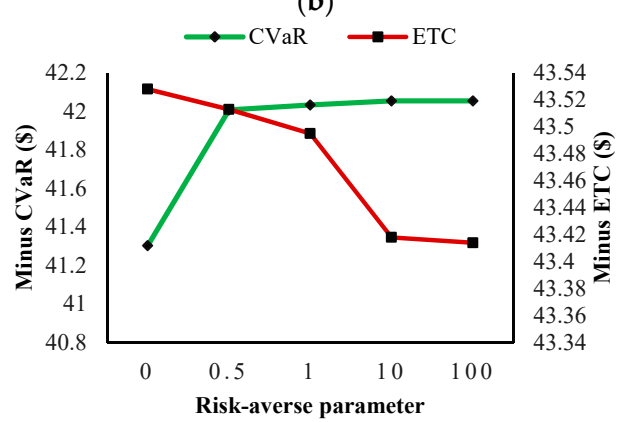

(d)

Figure 9. Sensitivity of ETC and conditional value at risk (CVaR) to the risk aversion parameter for each type of DRM: (a) Linear DRM; (b) Power DRM; (c) Logarithmic DRM; and (d) Exponential DRM.

Table 7. Sensitivity of output results of the MG operation problem to risk-aversion parameter in the fourth scenario.

\begin{tabular}{|c|c|c|c|c|c|c|c|c|c|c|c|c|}
\hline & \multicolumn{3}{|c|}{ Linear Model } & \multicolumn{3}{|c|}{ Power Model } & \multicolumn{3}{|c|}{ Exponential Model } & \multicolumn{3}{|c|}{ Logarithmic Model } \\
\hline & $\beta=0$ & $\beta=1$ & $\beta=100$ & $\beta=0$ & $\beta=1$ & $\beta=100$ & $\beta=0$ & $\beta=1$ & $\beta=100$ & $\beta=0$ & $\beta=1$ & $\beta=100$ \\
\hline$\sum_{t} P_{t}^{\text {grid-in }}(\mathrm{kW})$ & 642.650 & 654.603 & 655.569 & 677.780 & 689.734 & 690.700 & 666.785 & 678.739 & 679.705 & 657.579 & 669.533 & 670.499 \\
\hline$\sum_{t}^{t} P_{t}^{\text {grid-out }}(\mathrm{kW})$ & 352.385 & 352.385 & 350.823 & 290.973 & 290.973 & 289.410 & 313.450 & 313.450 & 311.887 & 323.568 & 323.568 & 322.005 \\
\hline$\sum_{t} P_{t, \mathrm{~s}}^{\mathrm{WT}}(\mathrm{kW})$ & 502.170 & 502.170 & 497.857 & 502.170 & 502.170 & 497.857 & 502.170 & 502.170 & 497.857 & 502.170 & 502.170 & 497.857 \\
\hline$\sum_{t}^{t} P_{t, s}^{\mathrm{PV}}(\mathrm{kW})$ & 174.372 & 174.372 & 174.372 & 174.372 & 174.372 & 174.372 & 174.372 & 174.372 & 174.372 & 174.372 & 174.372 & 174.372 \\
\hline$\sum_{t}^{t} P_{g, t, s}^{\mathrm{DG}}(\mathrm{kW})$ & 1322.038 & 1309.97 & 1311.20 & 1322.038 & 1309.97 & 1311.20 & 1322.038 & 1309.97 & 1311.20 & 1322.038 & 1306.97 & 1311.20 \\
\hline$\sum_{t}^{t} P_{t, s}^{\mathbf{E S}-\text { charge }}(\mathrm{kW})$ & 81.916 & 83.051 & 88 & 81.916 & 83.051 & 88.740 & 81.916 & 83.051 & 88.740 & 81.916 & 83.051 & 88.740 \\
\hline$\sum_{t, s} P_{t, \text { dcharge }}^{\mathrm{ES}}(\mathrm{kW})$ & 8.474 & 9.498 & 14.633 & 8.474 & 9.498 & 14.633 & 8.474 & 9.498 & 14.633 & 8.474 & 9.498 & 14.633 \\
\hline $\operatorname{ETC}(\phi)$ & 494.913 & -43.318 & -43.237 & 398.37 & -43.597 & -43.517 & 431.842 & -43.495 & -43.414 & 451.167 & -43.449 & -43.368 \\
\hline$\sum_{t} s\left(D_{t}^{\mathrm{DRM}}\right)(\phi)$ & -43.351 & -90.157 & -90.157 & -43.631 & -95.724 & -95.724 & -43.528 & -93.733 & -93.733 & -43.482 & -92.730 & -92.730 \\
\hline
\end{tabular}

\section{Conclusions and Future Works}

In this paper, a risk-based two-stage stochastic optimization approach is employed to model the operation problem of a grid-connected MG in the presence of DERs as well as the non-linear economic models of the incentive-based DRPs. Risk measurement is considered in the model to control uncertainties of the RERs as well as managing decision-making of the risk-averse MGO. The main conclusions from the proposed model obtained from numerical studies are as follows:

- The DRMs have various impacts on the MGL profile and the first-stage decisions of the MGO wherein the power model has the highest profitable model for the MGO.

- $\quad$ The risk-averse MGO and the risky MGO prefer the first-stage and the second-stage decisions in the proposed two-stage problem, respectively. 
- The risky MGO (risk neutral MGO) uses the whole capacity of RERs to meet its demand due to their low operation costs. On the other hand, the risk-averse MGO uses the lower output power of the RERs to control their uncertainties.

- In the presence of the MG, the energy mixes of an urban city ae changed so that Disco as the body responsible for supplying the energy consumption of the urban city can meet its required energy from the MG besides purchasing energy from the main grid.

The proposed approach in this paper to model the operation problem of MGs can be extended in future works as follows:

- Modeling the MG operation problem in cooperation with Disco considering the objective functions of both decision makers, i.e., Disco and the MG.

- Modeling the interaction between the MG with other decision makers in distribution networks including other MGs, retailers, various types of aggregators, and virtual power plants.

- Modeling several MGs in the form of an MG aggregator and its participation in the wholesale energy markets.

- The proposed model in this paper can be converted to a real-time application in which the operation problem of the MG can be modeled in the day-ahead and the real-time operation simultaneously. In this model, the stochastic optimization problem is changed so that the first-stage decision variables of the MGO, which are determined in the previous day of the operation, are purchased power from Disco and load interruption/shifting. The second-stage decision variables are determined considering the occurrence of the scenarios in real time, consisting of purchased power from Disco in real time, the power generation of DGs, and power charging/discharging of the battery.

Acknowledgments: J.P.S. Catalão acknowledges the support by FEDER funds through COMPETE 2020 and by Portuguese funds through FCT, under Projects SAICT-PAC/0004/2015-POCI-01-0145-FEDER-016434, POCI-01-0145-FEDER-006961, UID/EEA/50014/2013， UID/CEC/50021/2013， UID/EMS/00151/2013, and 02/SAICT/2017-POCI-01-0145-FEDER-029803, and also funding from the EU 7th Framework Programme FP7/2007-2013 under GA no. 309048.

Author Contributions: All authors have worked on this manuscript together, and all authors have read and approved the final manuscript.

Conflicts of Interest: The authors declare no conflict of interest.

\section{Nomenclature}

$\begin{array}{ll}\text { Acronyms } & \text { Conditional value at risk } \\ \text { CVaR } & \text { Distributed energy resources } \\ \text { DER } & \text { Distributed Generator } \\ \text { DG } & \text { Demand response } \\ \text { DR } & \text { Demand response model } \\ \text { DRM } & \text { Demand response program } \\ \text { DRP } & \text { Energy storage } \\ \text { ES } & \text { Micro-grid } \\ \text { MG } & \text { Micro-grid load } \\ \text { MGL } & \text { Price-based demand response } \\ \text { PBDR } & \text { Particle Swarm Optimization } \\ \text { PSO } & \text { Photovoltaic System } \\ \text { PV } & \text { Renewable energy resources } \\ \text { RER } & \text { Wind turbine } \\ \text { WT } & \end{array}$


Indices and sets

$\begin{array}{ll}g, G & \text { Index and set of DG } \\ s, S & \text { Index and set of scenario } \\ t, T & \text { Index and set of time period } \\ \text { Variables } & \end{array}$

DRM

$E_{t, s}^{\mathrm{ES}}$

$P_{t, s}^{\mathrm{ES}}$

$P_{t, s}^{\mathrm{ES}}$-charge

$P_{t}^{g r i d-i n}$

$P_{t}^{\text {grid-out }}$

$P_{t s}^{\mathrm{WT}}$

$P_{t, s}^{P V}$

$P_{g, t, s}^{\mathrm{DG}}$

$P_{g, t, s}^{\mathrm{ES}}$-dcharge

$S\left(D_{t}^{\mathrm{DRM}}\right)$

The amount of MGL considering each type of DRM (kW)

Energy storage of ES (kWh)

Power charging/discharging of ES $(\mathrm{kW})$

Power charging of ES (kW)

The amount of power purchased from main grid $(\mathrm{kW})$

The amount of selling power to main grid $(\mathrm{kW})$

The output power of WTs $(\mathrm{kW})$

The output power of PVs $(\mathrm{kW})$

The output power of DG $(\mathrm{kW})$

Power discharging of ES $(\mathrm{kW})$

Revenue from using DRPs $(\notin)$

$S_{1}\left(D_{t}^{\mathrm{DRM}}\right)$

$S_{2}\left(D_{t}^{\mathrm{DRM}}\right)$

$S_{3}\left(D_{t}^{\mathrm{DRM}}\right)$

Revenue from selling power to MGL after (and before) considering

DRPs $(\phi)$

Total incentive payment $(\phi)$

$u_{t}^{\text {grid }}$

Total penalty payment $(\phi)$

Binary variable (if equal to 1 ; MG purchases power from main grid and if equal 0, MG sells power to it)

$u_{t, s}^{\mathrm{ES}}$

Parameters

Binary variable (if equal to $1, \mathrm{ES}$ is charging and equal 0 for otherwise)

$A_{t}$

$C_{t}^{\text {grid-in }}$

$C_{t}^{\text {grid-out }}$

$C^{\mathrm{ES}}, C_{g}^{D G}$

$D_{t}^{\text {contract }}$

$D_{t}^{\text {initial }}$

Elasticity

$\underline{E}^{\mathrm{ES}}\left(\bar{E}^{\mathrm{ES}}\right)$

$\bar{P}^{\mathrm{ES}}$-charge

$\overline{\mathrm{P}}^{\mathrm{ES}-\text { dcharge }}$

$\bar{P}^{\text {grid-out }}$

$\bar{P}^{\text {grid-in }}$

$\bar{P}_{t s}^{\mathrm{WT}}$

$\bar{P}_{t, s}^{P V}$

$\bar{P}_{g}^{\mathrm{DG}}$

$P_{g}$
$\underline{P}_{g}^{\mathrm{DG}}$

$\mathrm{Pen}_{t}$

$\eta^{\mathrm{ES}}$

$\pi_{t}^{\text {initial }}$

$\pi_{t}^{P B D R}$

Incentive cost of DRPs ( $\varnothing$ )

Cost of power purchased from main grid $(\phi / \mathrm{kW})$

Revenue from selling power to main grid $(\phi / \mathrm{kW})$

Operation cost of ES and DGs $(\phi / \mathrm{kW})$

Value of demand assigned in the contract to be curtailed in DRPs $(\mathrm{kW})$

Initial demand level (kW)

Price elasticity of responsive load (\$)

Minimum (maximum) capacity of ES (kWh)

Maximum power charging of ES (kW)

Maximum power discharging of ES $(\mathrm{kW})$

Maximum selling power to main grid $(\mathrm{kW})$

Maximum purchased power from main grid $(\mathrm{kW})$

maximum WT output power $(\mathrm{kW})$

maximum PV output power $(\mathrm{kW})$

maximum DG output power $(\mathrm{kW})$

minimum DG output power $(\mathrm{kW})$

Penalty cost of DRPs in period $t(\phi)$

Efficiency of the ES

Initial price of electricity $(\phi / \mathrm{kWh})$

Electricity price in the price-based DRPs $(\phi / \mathrm{kWh})$

\section{References}

1. Palma-Behnke, R.; Benavides, C.; Lanas, F.; Severino, B.; Reyes, L.; Llanos, J.; Sáez, D. A Microgrid Energy Management System Based on the Rolling Horizon Strategy. IEEE Trans. Smart Grid 2013, 4, 996-1006. [CrossRef]

2. Baboli, P.T.; Shahparasti, M.; Moghaddam, M.P.; Haghifam, M.R.; Mohamadian, M. Energy management and operation modelling of hybrid AC-DC microgrid. IET Gener. Transm. Distrib. 2014, 8, 1700-1711. [CrossRef] 
3. Shen, J.; Jiang, C.; Liu, Y.; Wang, X. A Microgrid Energy Management System and Risk Management Under an Electricity Market Environment. IEEE Access 2016, 4, 2349-2356. [CrossRef]

4. Ou, T.-C.; Hong, C.-M. Dynamic operation and control of microgrid hybrid power systems. Energy 2014, 66, 314-323. [CrossRef]

5. Ou, T.-C.; Lu, K.-H.; Huang, C.-J. Improvement of transient stability in a hybrid power multi-system using a designed NIDC (Novel Intelligent Damping Controller). Energies 2017, 10, 488. [CrossRef]

6. Justo, J.J.; Mwasilu, F.; Lee, J.; Jung, J.-W. AC-microgrids versus DC-microgrids with distributed energy resources: A review. Renew. Sustain. Energy Rev. 2013, 24, 387-405. [CrossRef]

7. Ou, T.-C. A novel unsymmetrical faults analysis for microgrid distribution systems. Int. J. Electr. Power Energy Syst. 2012, 43, 1017-1024. [CrossRef]

8. Ou, T.-C. Ground fault current analysis with a direct building algorithm for microgrid distribution. Int. J. Electr. Power Energy Syst. 2013, 53, 867-875. [CrossRef]

9. Zhang, Y.; Gatsis, N.; Giannakis, G.B. Robust energy management for microgrids with high-penetration renewables. IEEE Trans. Sustain. Energy 2013, 4, 944-953. [CrossRef]

10. Li, Z.; Zang, C.; Zeng, P.; Yu, H. Combined two-stage stochastic programming and receding horizon control strategy for microgrid energy management considering uncertainty. Energies 2016, 9, 499. [CrossRef]

11. Ou, T.-C.; Su, W.-F.; Liu, X.-Z.; Huang, S.-J.; Tai, T.-Y. A modified bird-mating optimization with hill-climbing for connection decisions of transformers. Energies 2016, 9, 671. [CrossRef]

12. Aalami, H.A.; Moghaddam, M.P.; Yousefi, G.R. Demand response modeling considering Interruptible/Curtailable loads and capacity market programs. Appl. Energy 2010, 87, 243-250. [CrossRef]

13. Wu, K.; Zhou, H. A multi-agent-based energy-coordination control system for grid-connected large-scale wind-photovoltaic energy storage power-generation units. Sol. Energy 2014, 107, 245-259. [CrossRef]

14. Tazvinga, H.; Xia, X.; Zhang, J. Minimum cost solution of photovoltaic-diesel-battery hybrid power systems for remote consumers. Sol. Energy 2013, 96, 292-299. [CrossRef]

15. Nwulu, N.I.; Xia, X. Optimal dispatch for a microgrid incorporating renewables and demand response. Renew. Energy 2017, 101, 16-28. [CrossRef]

16. Han, Y.; Chen, W.; Li, Q. Energy Management Strategy Based on Multiple Operating States for a Photovoltaic/Fuel Cell/Energy Storage DC Microgrid. Energies 2017, 10, 136. [CrossRef]

17. Guo, L.; Liu, W.; Jiao, B.; Hong, B.; Wang, C. Multi-objective stochastic optimal planning method for stand-alone microgrid system. IET Gener. Transm. Distrib. 2014, 8, 1263-1273. [CrossRef]

18. Lee, S.; Jin, Y.; Jang, G.; Yoon, Y. Optimal bidding of a microgrid based on probabilistic analysis of island operation. Energies 2016, 9, 814. [CrossRef]

19. Gast, N.; Tomozei, D.-C.; Le Boudec, J.-Y. Optimal generation and storage scheduling in the presence of renewable forecast uncertainties. IEEE Trans. Smart Grid 2014, 5, 1328-1339. [CrossRef]

20. Talari, S.; Yazdaninejad, M.; Haghifam, M.R. Stochastic-based scheduling of the microgrid operation including wind turbines, photovoltaic cells, energy storages and responsive loads. IET Gener. Transm. Distrib. 2015, 9, 1498-1509. [CrossRef]

21. Aghajani, G.R.; Shayanfar, H.A.; Shayeghi, H. Demand side management in a smart micro-grid in the presence of renewable generation and demand response. Energy 2017, 126, 622-637. [CrossRef]

22. Huang, C.; Yue, D.; Deng, S.; Xie, J. Optimal Scheduling of Microgrid with Multiple Distributed Resources Using Interval Optimization. Energies 2017, 10, 339. [CrossRef]

23. Aalami, H.A.; Parsa Moghaddam, M.; Yousefi, G.R. Evaluation of nonlinear models for time-based rates demand response programs. Int. J. Electr. Power Energy Syst. 2015, 65, 282-290. [CrossRef]

24. Abdi, H.; Dehnavi, E.; Mohammadi, F. Dynamic economic dispatch problem integrated with demand response (DEDDR) considering non-linear responsive load models. IEEE Trans. Smart Grid 2016, 7, 2586-2595. [CrossRef]

25. Bahramara, S.; Parsa Moghaddam, M.; Haghifam, M.R. Modelling hierarchical decision making framework for operation of active distribution grids. IET Gener. Transm. Distrib. 2015, 9, 2555-2564. [CrossRef] 
26. Zakariazadeh, A.; Jadid, S.; Siano, P. Stochastic multi-objective operational planning of smart distribution systems considering demand response programs. Electr. Power Syst. Res. 2014, 111, 156-168. [CrossRef]

27. Uryasev, R.T.R.A.S. Optimization of conditional value-at-risk. J. Risk 2000, 2, 21-41.

28. Hatziargyriou, N.; Anastasiadis, A.G.; Vasiljevska, J.; Tsikalakis, A. Quantification of Economic, Environmental and Operational Benefits of Microgrids. In Proceedings of the PowerTech, 2009 IEEE Bucharest, Bucharest, Romania, 28 June-2 July 2009; pp. 1-8. [CrossRef] 